\title{
MANAGEMENT OF STREET ROUTING PROBLEMS USING DECISION SUPPORT SYSTEM
}

Servicing a large number of customers in a city zone is often a part of many logistics chains. The capacity of one delivery vehicle is limited, and at the same time, it usually serves a large number of customers. These problems are NP hard and often called a Street Routing Problem (SRP). Problems of this category are similar to the problems named as Vehicle Routing Problem (VRP). In this paper, these problems are explored and potential solution paths for their management are described. As presented in the paper, the management of street routing problems could be effectively done only by using decision support systems or other informatics systems, such as Geographical Information Systems (GIS).

\section{Introduction}

Street Routing Problems (SRP) as a problem of servicing a large number of customers in a city zone is often a part of many logistics chains. Into the SRP category we can include problems like newspaper delivery to the corner shops, waste removal, postal service delivery, commercial freight delivery, and meter reading.

Practical experience from the solution of the SRP in the Slovak Republic suggests that they are in most cases solved by an expert's experienced judgment. The main problem of SRP is that the number of customers is large and the number of delivery path combinations is enormous. It is not possible for a human to explore even a fragment of all these combinations. Experts usually take the first acceptable solution, which may be far from the optimal solution. As the experimental results show in the case of SRP, the error on the length of delivery routes based on an expert's judgment when compared to optimal solution is in the range of $10 \%-25 \%$.

The SRP is in many cases similar to the classical Vehicle Routing Problem (VRP). There are some important differences between the SRP and VRP.

- The number of customers in the SRP is large compared to the VRP. In the VRP the number of customers is in the hundreds, and in the SRP, it could be hundreds of thousand.

- The service time for one customer in the SRP is short and the route path for one vehicle contains several hundreds of these customers.

- The density of the street network in the SRP is high compared to the regular road network in the VRP.

- The distance between two close customers in the SRP is small and it is possible to get from one customer to another customer through a small number of possible network connections.

- Traffic regulations in the city zone are more complicated and more restrictive than traffic regulations in a regular road network. These regulations could greatly affect the expense for the routes.
- The access points to the customers are important and have a large effect on the total cost.

Formerly, dispatchers could communicate with the drivers of the service vehicles only using radio stations. Nowadays, they can also use other technologies, such as mobile phones, palmtops connected to the wireless internet, and GPS together with GIS map databases. In these days they could assign jobs for each vehicle, even outside of the dispatching center, as well as help navigate the driver in an unknown area.

\section{Solution of SRP using VRP techniques}

As shown in the previous section, there are some common properties between the SRP and VRP and some differences between them. In 2005, we explored a possible use of VRP techniques for solving SRP instances [4]. We were solving the problem of postal service in the city of Galanta. Customers in the postal deliveries usually are each house or doors in the large blocks. Customers are usually located on both sides of the street segments and could be serviced differently depending on local conditions and other regulations. In Figure 1 there are three typical cases.

As our experiments show, applying VRP techniques on these SRP cases directly, that means taking each customer in the SRP as a customer of the VRP, the results are usually not acceptable. Observe in Figure 2 that customers on one street segment are serviced by two vehicle routes.

As the result of our experiments, we can say:

- SRP solutions take more time than typical VRP solutions because of local restrictions, combined with the number of customers in the typical SRP.

- To be able to compute SRP in most cases, number of customers should be decreased by their aggregation.

\footnotetext{
* Peter Matis

Department of Transportation Systems, Faculty of Management Sciences and Informatics, University of Zilina, E-mal: Peter.Matis@fria.utc.sk
} 


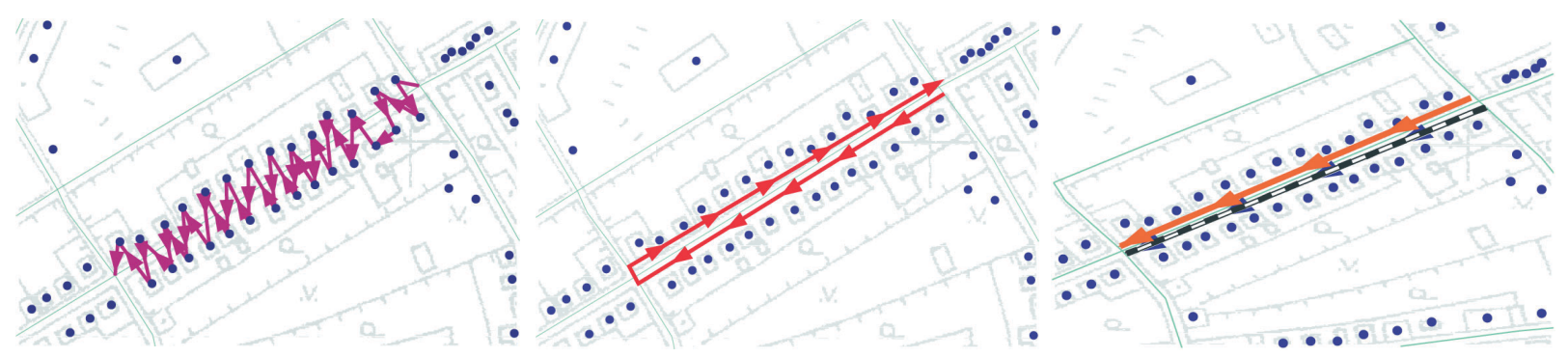

Fig. 1 Cases of service on the street segments

- Expenses to maintain customer and street network databases are high.

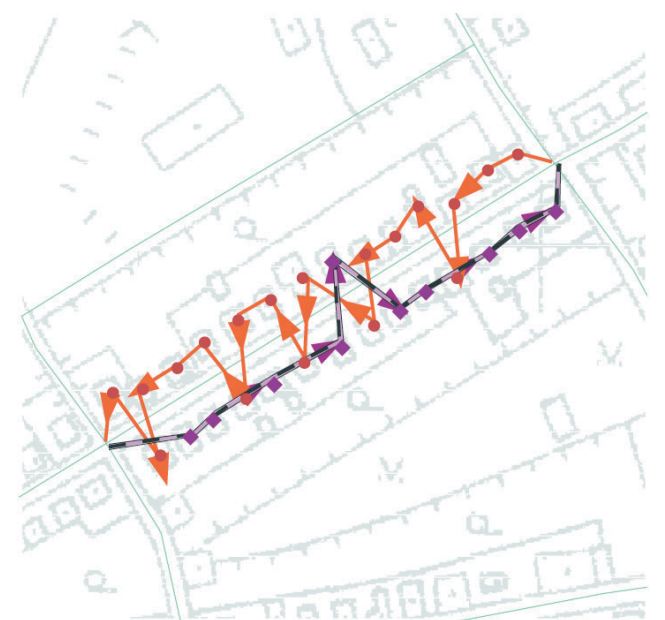

Fig. 2 Solutions of SRP using VRP strategies are not acceptable

One of the important measures for a good solution of the SRP is the parameter that tells us about the balancing of routes. It is almost always important to balance the routes, so that there are no cases when one postman has to always work overtime while another postman is using only part of his or her capacity. As presented in Tables 1 and 2, using the proper algorithm for the SRP solution, we were able to balance postal routes. By using real street networks, the average traveling speed and measured service times, we were able to decrease the number of routes in the city of Galanta by two. These results also show that no route lasts longer then 285 minutes, which was our limit. This is 48 minutes less than the longest route in the current situation. The difference in time between the shortest and the longest route is 93 minutes.

\section{Customer aggregation}

Practical experiments show that if we want to apply the VRP heuristics to the solution of the SRP, we need to aggregate customers to be able to solve the problem. One of the purposes for doing it is the fact that computational time expands with large numbers of customers and, by aggregation we keep this number in a range that is comparable to the typical VRP.
Current postal routes in Galanta

Tab. 1

\begin{tabular}{|c|c|c|}
\hline Route & Length [m] & Total time [min] \\
\hline I & 8367 & 277 \\
\hline II & 9881 & 271 \\
\hline III & 12378 & 333 \\
\hline IV & 8985 & 233 \\
\hline V & 5969 & 182 \\
\hline VI & 5815 & 233 \\
\hline VII & 12533 & 257 \\
\hline VIII & 9506 & 302 \\
\hline IX & 11715 & 307 \\
\hline X & 6888 & 326 \\
\hline XI & 6037 & 268 \\
\hline XII & 4403 & 172 \\
\hline
\end{tabular}

Computed postal routes in Galanta

Tab. 2

\begin{tabular}{|c|c|c|}
\hline Route & Length [m] & Total time [min] \\
\hline A & 7200 & 283 \\
\hline B & 12450 & 279 \\
\hline C & 6110 & 282 \\
\hline D & 3570 & 284 \\
\hline E & 6160 & 285 \\
\hline F & 5020 & 283 \\
\hline G & 8145 & 255 \\
\hline H & 5972 & 266 \\
\hline I & 9240 & 285 \\
\hline J & 5380 & 192 \\
\hline
\end{tabular}

- In distance aggregation, we aggregate customers based on their distance. It is possible to aggregate customers that are located on different street segment(s).

- Line aggregation allows aggregation only of customers that are located on the same side of one street segment.

- Line-segment aggregation is the special case of line aggregation where all customers on one side of one street segment create one aggregate.

- Address aggregation allows the combination of customers that have similar address. 
Properties of aggregations have been explored using the following experiment. Settlements in Slovakia were chosen, and divided into 5 categories. For each settlement there was a known street network and location of customers. For the line and the distance aggregation, the maximum distance between two customers in the aggregate was set to $80 \mathrm{~m}$. In all the cases, we have set customer delivery time to $10 \mathrm{sec}$ and requested quantity to 2 units. We controlled the total time for the delivery inside of the aggregate area and limited it to 10 minutes and total requested units to 100 . We can define the total delivery time as:

$$
t_{\text {aggregate }}=\left(\sum_{i \in \text { aggregate }} t_{i}\right)+\max _{i, j \in \text { agreggate }}\left(c_{i j}\right) / v
$$

Where is a service time for customer $i, v$ is the average traveling vehicle speed (in our experiment, it was set as $4.5 \mathrm{~km} /$ hour) and $c_{i j}$ is a network distance between customers $i$ and $j$. For a better evaluation, an estimation could be used based on the length of the traveling salesman problem for all of the customers in the aggregate. Table 3 presents these times.

Experiments have show that the best aggregation is distance aggregation. On the other hand distance aggregation shows high deformation of aggregated positions against the original positions of customers.

\section{Transformation of Arc Routing Problems to Node Routing Problems}

Many SRPs could be viewed as Arc Routing Problems (ARP). There is a special case of ARP called the Capacitated Arc Routing Problem (CARP) [3], [1]. We present the definition on the graph. Let $G=(V, E)$ be an undirected graph with a set $V$ of $n$ nodes and a set $E$ of $m$ edges. A fleet of identical vehicles of capacity $Q$ is based at a depot node $S$. A subset of $r$ edges $R \subset E$, denoted as required edges, require service by a vehicle. Any edge can be traversed any number of times. Each edge $(i, j)$ has a traversal cost $c_{i j}$ and a demand $q_{i j}$. The goal is to determine a set of vehicle trips (routes) of minimum total cost, so that each trip starts and ends at the depot, each required edge is serviced by one single trip, and the total demand handled by any vehicle does not exceed $Q$.

CARP is an NP hard problem and currently there does not exist any optimal algorithm to solve cases of a realistic size. There are heuristics to solve real size CARPs, their performance is in many cases not acceptable [2]. One possible solution of this problem is to transform a CARP to a capacitated vehicle routing problem (CVRP). There are several good heuristics that perform well on realistic size problems.

Let's transform edge $(i, j) \in R$ into the nodes $s_{i j}$, $s_{j i}$, which represents edge's end points. The CVRP is then defined on the complete graph $H=(N, A)$, where the set of the nodes $N$ is defined:

$$
N=N^{\prime} \cup\{S\}=\bigcup_{(i, j) \in R}\left\{s_{i j}, s_{j i}\right\} \cup\{S\}
$$

Demand in the new nodes is defined as:

$q\left(s_{i j}\right)=q\left(s_{j i}\right)=\frac{1}{2} q(i, j)$

Distances in $H$ are defined as:

$$
d\left(s_{i j}, s_{k l}\right)= \begin{cases}0 & (i, j)=(k, l) \\ c_{i j} & (i, j)=(l, k) \\ s_{-} \text {path }(i, k) & (i, j) \neq(k, l) ;(i, j) \neq(l, k)\end{cases}
$$

\begin{tabular}{|c|c|c|c|c|c|c|c|c|c|}
\hline Cat & NCS & TNC & ANC & Agreg & NA & ATA & MTA & MeTA & SD \\
\hline \multirow{3}{*}{$400-4000$} & \multirow{3}{*}{75} & \multirow{3}{*}{32150} & \multirow{3}{*}{428} & DIST & 8167 & 60.2 & 212 & 56 & 44.7 \\
\hline & & & & LINE & 12096 & 41 & 183.9 & 35.5 & 32.2 \\
\hline & & & & SEG & 5285 & 130.6 & 600 & 77 & 158.7 \\
\hline \multirow{3}{*}{$4000-10000$} & \multirow{3}{*}{2} & \multirow{3}{*}{2035} & \multirow{3}{*}{1017} & DIST & 1165 & 45.7 & 185.1 & 36.6 & 40.5 \\
\hline & & & & LINE & 1495 & 35.4 & 183.9 & 10 & 31.4 \\
\hline & & & & SEG & 579 & 141.5 & 600 & 65.4 & 188.4 \\
\hline \multirow{3}{*}{30000} & \multirow{3}{*}{1} & \multirow{3}{*}{1616} & \multirow{3}{*}{1616} & DIST & 429 & 65 & 218 & 61.5 & 47.8 \\
\hline & & & & LINE & 680 & 41.5 & 147.7 & 35.4 & 31.5 \\
\hline & & & & SEG & 402 & 87 & 600 & 58.2 & 97.3 \\
\hline \multirow{3}{*}{\multicolumn{4}{|c|}{ Average }} & DIST & 4254 & 57.3 & 220.8 & 51.2 & 44.5 \\
\hline & & & & LINE & 6264 & 39.8 & 184.2 & 29.9 & 32.6 \\
\hline & & & & SEG & 2738 & 120.4 & 600 & 67.9 & 147.3 \\
\hline
\end{tabular}

$s_{\_}$path $(i, j)$ represents the shortest path between the nodes $i$ and $j$ on the original graph $G$. Transformation is presented in figure 3.

Cat - Category by number of habitants in cities, NCS - Number of cities in the sample, TNC - Total number of customers, ANC - Average number of customers in city, Agreg - Agregation, NA - Number of aggregates, ATA - Average time to serve aggregate, MTA - Maximum time to serve aggregate, MeTA - Median time to serve aggregate, SD - Standard deviation, DIST - Distance aggregation, LINE - Line aggregation, SEG - Linesegment aggregation 

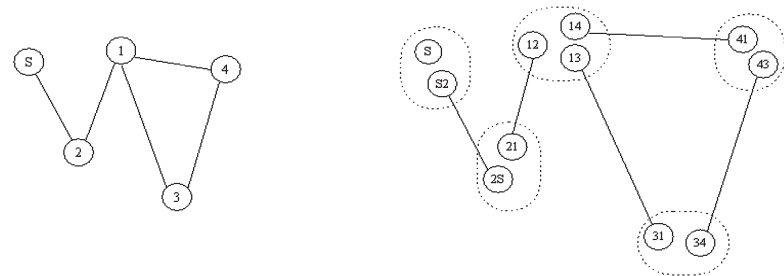

Fig. 3 Transformation of CARP to CVRP

Finally, we require that all edges $\left\{\left(s_{i j}, s_{j i}\right) \mid(i, j) \in R\right\}$ belong to the solution, i.e. we only accept CVRP solutions where $s_{i j}$ and $s_{j i}$ are visited in sequence, either $s_{i j} \rightarrow s_{j i}$ or $s_{j i} \rightarrow s_{i j}$. The transformed graph has $2 * r+1$ nodes, and we expect that fixing these edges in the CVRP can make an algorithm perform almost as if on an instance with $r$ nodes. [6]

\section{Interactive methods and decisions support systems}

Recent development in the real street routing problems shows that there is a need to make routing software a part of a larger system [7]. One of the possible solutions to this is to integrate routing software within GIS [5]. GIS can be helpful in the collection, storing and management of large geographical databases used in the routing software - i.e. transportation network databases and databases of customers. GIS can be also used for creation of all outputs from the routing software, including detailed maps for each route with the route description. One of the key properties of GIS for use with the routing software is the interactive and userfriendly environment. This is valuable for many real SRP, because as we have found out, there is always a need to work in a co-operation of expert-routing software. Routing software can find a good solution and explore the possibilities and an expert can change calculated routes to explore other possibilities based on the expert's judgment.

GIS have several useful features that could help improve routing software performance. To mention only a few here - we may pay attention to the capabilities as safe database management, flexible symbols, map management, drawing capabilities, safety and interoperability.
Decisions support systems (DSS) are important for solving real SRPs. One use of the routing software results is not only a solution of the problem, but also the ability to explore several other possibilities, or perform operations like exchanging parts of two routes or seeing how changes in the transportation network, regulations or policies could affect the routes, expenses and other parameters. Currently there exist two trends in the development of DSS for SRP.

- Independent software packages specialized for SRP with limited amount of DSS capabilities. Here we can note software like GeoRoute from the Canadian software firm GIRO. It is not an open system. It is an expensive product that costs 1,000,000 EUR for one installation. As an example of other such systems, we can use TRANSCAD created in the US, which has some similarities to GeoRoute. GeoRoute is more feasible for solving the SRP because it is specialized for the street routing. TRANSCAD is more specialized in the node routing.

- Integrated systems based on the GIS or CAD. As an example, we can put ArcView with its ArcGIS Network Analyst. It is specialized in the node routing. The user can implement extension and then make it an integral part of the whole system.

As presented here, these DSS are usually not appropriate for use with real SRP or they are expensive for use in middle size organizations that operate in cities with operations based on the routing. One can find it to be a good solution to use GIS and integrate routing software and DSS inside of GIS.

\section{Conclusion}

Paper summarizes the management of street routing problems. Management of the SRP is a new field of research and it is growing. There are expanding activities in the cities that can be presented as street-based tasks. Unfortunately, there is not a good methodology for solving the general SRP. A potential solution to the management of SRP is the integration of routing software with GIS and DSS. On of the possible solution for this problem is to aggregate the large number of customers into the clusters and then solve VRP. Paper shows characteristics of three different types of aggregations and shows possible development for future.

\section{References}

[1] AMPONSAH, S. K., SALHI, S.: The investigation of a class of capacitated arc routing problems: the collection of garbage in developing countries, Waste Management 24, 2004, p. 711-721.

[2] BEULlENS, P., MUYLDERMANS, L., CATTRYSSE, D., OUDHEUSDEN, D.: A guided local search heuristic for the capacitated arc routing problem, European Journal of Operational Research 147, 2003, p. 629-643.

[3] GOLDEN, B. WONG, R. T.: Capacitated arc routing problems, Networks 11, 1981, p. 305-315.

[4] JANÁČEK, J.: Optimization on transportation networks, EDIS-ŽU, Žilinská univerzita, 2003, p. 248.

[5] LONGLEY, P., BATTY, M.: Spatial analysis: Modelling in a GIS environment. John Wiley \& Sons, Inc., New York, 1996, ISBN 0-470-23615-9.

[6] PEARN, W., ASSAD, A., GOLDEN, B.: Transforming Arc Routing into Node Routing Problems, Computers \& Operations Research 14(4) 1987 p. 285-288.

[7] RUIZ, R., MAROTO, C., ALCARAZ, J.: A decision support system for a real vehicle routing problem, European Journal of Operational Research 153, 2004, p. 593-606. 